D.O.I.: $10.3895 / \mathrm{S} 1808-04482007000200009$

\title{
MODELOS DE ANÁLISE DE AGLOMERADOS INDUSTRIAIS: IMPLICAÇÕES NO ESTUDO DO AGLOMERADO DE PETRÓLEO E GÁS DO NORTE FLUMINENSE
}

\section{MODELS OF ANALYSIS TO INDUSTRIAL AGGLOMERATIONS: IMPLICATIONS ON THE STUDY OF THE NORTH FLUMINENSE OIL AND GAS AGGLOMERATION}

\author{
Bruno dos Santos Silvestre ${ }^{1}$; Paulo Roberto Tavares Dalcol ${ }^{2}$ \\ ${ }^{1}$ Centrais Elétricas Brasileira S.A. - ELETROBRÁS - Rio de Janeiro - Brasil \\ bruno.silvestre@eletrobras.com \\ ${ }^{2}$ Pontifícia Universidade Católica do Rio de Janeiro - PUC-Rio - Rio de Janeiro - Brasil \\ prtd@ind.puc-rio.br
}

\begin{abstract}
Resumo
Com base nas crescentes mudanças tecnológicas e gerenciais da atualidade, as empresas buscam meios de alcançar vantagens competitivas, de inovar e se manter em atividade no mercado de forma sustentada. Com o intuito de discutir as novas formas de inserção de empresas na dinâmica econômica atual, o presente artigo analisa, no contexto das abordagens de arranjos produtivos locais (APL's), dos keiretsu japoneses, dos clusters e dos sistemas setoriais de inovação (SSI), pontos positivos e negativos dessas configurações, quando utilizados na análise do aglomerado de petróleo e gás do Norte Fluminense. O objetivo é identificar a aplicabilidade dos conceitos e abordagens de APL, keiretsu de produção (modelo de produção japonês) cluster e sistemas setoriais de inovação ao aglomerado de petróleo e gás do Norte Fluminense. Além disso, busca-se a confrontação das peculiaridades de alguns estudos empíricos de aplicação desses conceitos com o aglomerado em questão.
\end{abstract}

Palavras-chave: Inovação; Aglomerados industriais; Indústria de petróleo e gás.

\section{Introdução}

A globalização da economia vem trazendo um aumento da competição entre as empresas e a diminuição de fronteiras físicas, bem como a aproximação entre mercados produtores e consumidores, obrigando as organizações que desejam se manter ativas e ter sucesso a procurarem novas alternativas de processos, métodos, produtos e gestão.

Tais mudanças e, principalmente, a desverticalização das empresas, focando, nos dias de hoje, apenas as competências essenciais do negócio (core competencies) e não mais todas as etapas do processo produtivo, fazem com que as empresas tenham que optar por diferentes estratégias em 
qualquer setor industrial. As empresas deixariam, então, de tentar competir diretamente com as empresas líderes e passariam a compor uma rede de fornecedores inserida no mercado global, alcançando grande importância no cenário econômico industrial.

Essas transformações geram ganhadores e perdedores. As empresas que não se adaptarem às responsabilidades, às novas formas de competição e à flexibilidade nesse ambiente dinâmico tendem a morrer. A empresa que optar por ser fornecedora de empresas 'âncoras', aproximando-se das mesmas, deve especializar-se e direcionar seus negócios para aquele segmento específico, embora corra o risco de perder seu poder de barganha ao longo do tempo.

Esta tendência faz com que as empresas se aglomerem em determinadas áreas, as quais chamamos de aglomerados industriais, para que possam se aproximar das empresas líderes, alcançar novas vantagens competitivas, inovar em parceria com outras firmas e ganhar espaço nesse novo ambiente, competindo diretamente por mercados e tirando proveito da proximidade para incrementar a interação, a cooperação e a troca de conhecimentos com outras firmas. Tal postura é fundamental para as firmas que desejam buscar a sustentabilidade a longo prazo, pois o sucesso empresarial atualmente passa, necessariamente, por dois conceitos fundamentais: cooperação e inovação, os quais não se pode implementar isoladamente.

As aglomerações de firmas podem ser, em seu turno, de duas naturezas distintas: as que são baseadas na cultura e na tradição existente na região onde se encontram e as baseadas em algum tipo de oportunidade específica existente na região (recursos naturais por exemplo). O primeiro tipo está mais intimamente ligado à essência e à história da região, a qual possui experiência na comercialização dos produtos, possui certo reconhecimento como produtora dos mesmos, possui mão-de-obra específica, entre outras características que contam positivamente na atração das empresas. Já o segundo tipo de aglomeração se sustenta enquanto existir a vantagem gerada pela disponibilidade dos recursos (naturais ou humanos).

Diversas abordagens são utilizadas para analisar essas aglomerações de empresas. A enorme gama de conceitos existentes na literatura faz com que certa confusão emerja dentro da área de conhecimento. Cada uma dessas abordagens apresenta peculiaridades e são mais convenientemente aplicadas a determinados casos.

Nesse contexto, abordaremos o aglomerado do setor de petróleo e gás do Rio de Janeiro, mais especificamente do Norte Fluminense (Campos e Macaé), buscando a aplicabilidade dos conceitos e abordagens de arranjo produtivo local (APL), dos keiretsu de produção japoneses, de clusters e de sistemas setoriais de inovação (SSI) em tal aglomerado. Para isso, apontamos dois exemplos práticos para efeito de comparação: o cluster industrial da Embraer em SP e o sistema setorial de inovação de máquinas e ferramentas, além do modelo Toyota de produção e do APL calçadista do Vale dos Sinos - RS. 


\section{O aglomerado de petróleo e gás do Norte Fluminense}

A Petrobrás atingiu no primeiro trimestre de 2005 o seu recorde de produção, com uma média diária de 2,071 milhões de barris (RANGEL, 2005). A principal província petrolífera do país, a Bacia de Campos, é responsável por mais de $80 \%$ da produção de petróleo e quase $50 \%$ do gás produzido no país. Segundo a Petrobrás, a Bacia de Campos possui, atualmente, 39 campos de exploração de petróleo, com um ativo de 41 unidades de exploração (sendo 14 plataformas fixas, 16 plataformas flutuantes e 11 navios-plataformas). Possui ainda quase 170 embarcações na bacia, mais de $4.000 \mathrm{Km}$ de dutos de óleo e gás natural, além de infra-estrutura de apoio (helipontos, ancoradouros, unidades em terra, entre outros). A escala em que atua a empresa na Bacia de Campos não encontra comparativo em nenhuma região e com nenhuma outra empresa no mundo. São cerca de 6.900 empregados diretos e mais 28.000 empregados de empresas terceirizadas trabalhando dentro de instalações Petrobrás. A empresa estima que as atividades petrolíferas na Bacia de Campos gerem cerca de 100.000 empregos indiretos em todo o estado do Rio de Janeiro. Com as operações de exploração e produção (E\&P) de petróleo e gás na Bacia de Campos, a Petrobrás conquistou uma posição de liderança mundial em tecnologia de águas profundas, exportando a tecnologia de exploração e produção nesses ambientes para vários países.

A importância da Bacia de Campos e, conseqüentemente, do estado do Rio de Janeiro para a indústria de petróleo nacional é notória. Destaca-se que a maioria das atividades de exploração e produção do setor no Rio de Janeiro está concentrada na região Norte Fluminense (Campos e Macaé).

Somente em Macaé existem atualmente cerca de 400 empresas fornecedoras de produtos e serviços em atividades offshore. No total, são cerca de 1.400 empresas fornecedoras dos mais diversos produtos e serviços, como sistemas complexos de alta tecnologia em perfuração e produção de petróleo, de mergulho, de geoprocessamento, de meio-ambiente, de material de escritório, de roupa de cama e de alimentação e segurança para as unidades de exploração no mar, entre outras (SILVESTRE e DALCOL, 2004).

Pode-se perceber que o aglomerado de empresas do setor de petróleo do Rio de Janeiro consiste de uma estrutura bastante robusta, verticalizada e com a presença de empresas fornecedoras da quase totalidade dos produtos e serviços utilizados na cadeia produtiva do setor de exploração e produção $(\mathrm{E} \& \mathrm{P})$ de petróleo e gás. A Petrobrás exerce o papel de empresa âncora (com papel de comando), consumidora direta ou indireta de todos esses produtos e serviços, produzidos e oferecidos por uma imensa rede de subcontratação composta por pequenas, médias e grandes empresas nacionais, além de transnacionais de portes variados. 


\section{A abordagem de APL, dos keiretsu japoneses de produção e casos empíricos}

$\mathrm{Na}$ estrutura dos keiretsu, todas as firmas são classificadas por níveis de fornecimento e responsabilidade (onde no primeiro nível, em contato direto com a empresa âncora, estão os fornecedores de produtos mais complexos). A presença da empresa âncora (a chamada empresamãe) é fundamental para as relações de governança e de poder dentro do sistema, podendo manter relações de negócios com as empresas subcontratadas por décadas (GERLACH, 1992; CELESTE, 1993; MASIERO, 1995; AMATO NETO, 2000). Para Lastres \& Cassiolato (2000), a estrutura dos keiretsu é vista como uma forma flexível, estável e competitiva de organização empresarial, permitindo às empresas explorarem de forma mais completa as vantagens da colaboração.

O caso da Toyota japonesa apresenta uma característica fundamental: a presença de um grande banco dentro da estrutura que fornece o crédito necessário para o desenvolvimento das firmas associadas e, principalmente, da empresa-mãe. A parceria e confiança são elementos predominantes nas relações de negócio. Da mesma forma, a estrutura dos keiretsu é organizada de modo que um fornecedor não se sinta ameaçado pelo outro, evitando que cada um busque preços cada vez menores, reduza suas margens de lucro sem diminuição de custos, gerando um bloqueio no fluxo horizontal de informações, impedindo os avanços técnicos e a melhoria da qualidade dos produtos fornecidos. Os fornecedores da Toyota eram companhias independentes, com contabilidade autônoma que, no entanto, estavam intimamente envolvidos no desenvolvimento dos produtos, compartilhando seus destinos com a Toyota (WOMACK et al., 1992).

De acordo com o aspecto estrutural, pode-se ressaltar semelhanças entre o aglomerado de petróleo e gás do Norte Fluminense e modelo da Toyota, principalmente no que diz respeito à estrutura de comando liderada por uma única empresa, o que leva a uma configuração bastante verticalizada. No entanto, existem profundas diferenças entre as duas configurações, sobretudo na existência de crédito próprio para as empresas pertencentes ao keiretsu (presença de um banco na estrutura) e a relação entre a empresa-mãe e os fornecedores que, no modelo Toyota, apresenta um sentido de parceria muito mais intenso. No aglomerado de petróleo e gás o ambiente entre fornecedores é bastante competitivo (longe da parceria existente nas estruturas japonesas), com pouca ou nenhuma cooperação horizontal entre fornecedores e grande proteção aos aspectos industriais (segredos industriais), no qual os pequenos fornecedores possuem pouco poder de barganha junto à Petrobrás, o que não ocorre com os grandes fornecedores transnacionais. Essas características são em parte agravadas pela presença de atores globais, pela importância estratégica do conhecimento, da tecnologia e da inovação.

Já em relação à abordagem dos arranjos produtivos locais (APL's), é necessário dispensar alguma atenção para a diferença entre dois conceitos encontrados na literatura que podem gerar 
dúvidas: sistemas e arranjos. Sistemas e arranjos são classificados em termos relativos. Essa situação ocorre em virtude de ser impossível se estabelecer, no mundo real, um modelo ideal de aglomerado. Podemos determiná-los, mais especificamente, de acordo com definição sugerida em Cassiolato e Szapiro (2002), conforme se segue:

- Por um lado, um arranjo se refere a uma aglomeração produtiva cujas interações entre agentes locais não são desenvolvidas de modo que se caracterize esse arranjo como um sistema (não existe um grau de articulação significativo entre as empresas). Fica explícito que as estruturas de arranjos apresentam uma configuração sem suficientes conexões com o ambiente e fluxos predominantemente de bens e serviços;

- Por outro lado, um sistema se refere a aglomerações de agentes econômicos, políticos e sociais, localizados no mesmo território, apresentando conexões de articulação consistentes, interação, cooperação e aprendizado, buscando a introdução de novos produtos e processos (de uma forma dinâmica e com alto grau de articulação). Cabe ressaltar que os sistemas são estruturas conectadas (interna e externamente), apresentando fluxos baseados não somente em produtos e serviços, mas também em conhecimento;

O caso empírico estudado sobre a abordagem de APL é o desenvolvido na região do Vale dos Sinos, no Rio Grande do Sul. O APL conta com cerca de 500 empresas produtoras de calçados que atuam principalmente no nicho de mercado de calçados femininos de couro (apesar de existirem diversas empresas que atuam na produção de calçados masculinos e infantis). Este APL é responsável por mais de 80\% das exportações brasileiras de calçados. (VARGAS e ALIEVI, 2000). No entanto, as estruturas estudadas, utilizando a abordagem de APL, geralmente possuem uma governança mais pulverizada, i.e. estrutura menos verticalizada (SILVESTRE e DALCOL, 2003) do que a estrutura encontrada no aglomerado de petróleo e gás do Norte Fluminense.

O modelo de abordagem de APL's possui uma aplicabilidade muito mais próxima das estruturas horizontais (onde a liderança é exercida por mais de uma empresa, sem uma firma única com papel central no sistema). Geralmente, a estrutura onde se aplica esta abordagem apresenta um grande número de empresas de micro, pequeno e médio porte, nas quais a presença de atores globais é bastante limitada, como por exemplo o aglomerado de rochas do Espírito Santo (VILLASCHI e SABADINI, 2000), o coureiro calçadista de Nova Serrana (CROCCO et al, 2001), o coureiro calçadista do Vale dos Sinos (VARGAS e ALIEVI, 2000) e o de confecções de Nova Friburgo (LA ROVERE, et al, 2000). 


\section{A abordagem de clusters e o aglomerado aeronáutico de São José dos Campos}

O conceito de cluster está relacionado a um aglomerado de firmas em um ou mais setores correlatos ou indústrias complementares (não necessariamente na mesma indústria ou setor), geograficamente concentradas. Pesquisadores, em estudos relacionados a cluster, geralmente dão especial atenção à proximidade territorial, com a qual as firmas operam. Estes aglomerados situamse no mesmo espaço geográfico que pode estar na mesma cidade, região, estado ou em um mesmo país.

Para Schmitz, cluster industrial "é definido como a concentração geográfica e setorial de firmas" (SCHMITZ, 1997: 3). Fica claro, contudo, que um cluster industrial possui uma característica setorial que é muito importante nas definições e na análise em que se utiliza esta abordagem.

As dimensões analisadas na abordagem de cluster possuem elementos relacionados à produção, segundo os quais, as conexões são principalmente baseadas em fluxos de bens e serviços, compondo os sistemas de produção (SCHMITZ, 1999 e PORTER, 1998). Do mesmo modo, algumas análises destacam as características relacionadas ao conhecimento, aprendizagem e inovação (SAXENIAN, 1994 e BELL e ALBU, 1999). Tais autores enfatizam a importância dos sistemas de conhecimento (os fluxos que se baseiam na troca de conhecimento) e mostram preocupação com a inovação e a sustentabilidade a longo prazo.

No entanto, com base na literatura mais recente de cluster (MALMBERG, 2003; BEAUDY e BRESCHI, 2003; entre outros), a atividade econômica agrupada de um setor particular por si só não representa nenhuma vantagem para as firmas.

Para Malmberg (2003: 159), "o desafio, se desejamos entender como um milieu pode explorar e sustentar a competitividade internacional, é analisar como tais milieus podem desenvolver conexões para controlar as fontes de conhecimento especializado, onde quer que estejam no mundo".

O caso empírico descrito neste trabalho é o do aglomerado aeronáutico de São José dos Campos. O modelo de organização industrial no aglomerado aeronáutico pode ser mais bem compreendido pela concepção de uma configuração arquitetural de redes centralizadas (core networks): onde um conjunto formado por cerca de 40 empresas de médio e pequeno porte organizadas em torno da economia gerada por uma firma, a Embraer. As relações interfirmas desenvolvidas na região apresentam níveis diferenciados de integração, fluxos de transação comercial e tecnológicos, mas com um alto grau de coordenação. Trata-se de uma organização industrial interempresas, de caráter hierarquizador; um tipo de "sistema solar": "empresa-mãe" e suas satélites subcontratadas, fundamentadas em um intercâmbio particular entre fornecedores e 
prestadores de serviços de engenharia, administrativos e produtivos, formados por pequenas e médias empresas (BERNARDES, 2000).

Cabe ressaltar que, nesta configuração estrutural que circunda a Embraer, a presença de empresas de médio e pequeno porte é bastante significativa. Existem parcerias com empresas de porte maiores, mas estas na maioria das vezes se encontram fora do aglomerado. Essa configuração traz a possibilidade de parcerias locais mais coesas, em virtude da pouca presença de grandes atores globais e da competição moderada entre fornecedores similares. Apesar disso, a estrutura de liderança exercida por uma grande empresa se assemelha bastante com a estrutura encontrada no aglomerado de petróleo e gás do Norte Fluminense, no qual a Petrobrás exerce o papel de líder da rede de subcontratação.

Da mesma forma que o modelo Toyota, o cluster aeronáutico da Embraer não apresenta características específicas de um APL (BERNARDES, 2000). Isso se deve a sua estrutura verticalizada, na qual uma empresa - a Embraer - exerce o papel central na estrutura de comando. Assim como os APL's, este aglomerado apresenta também forte conteúdo vocacional e histórico em seu desenvolvimento. Além disso, diferentemente do aglomerado de petróleo e gás do Rio de Janeiro, podemos ressaltar a pequena presença de atores globais e rede de subcontratação baseada na presença de micro, pequenas e médias empresas.

\section{5 - A abordagem de SSI e o caso do setor de máquinas e ferramentas}

De acordo com Malerba (2004a), esta abordagem foca a inovação em um setor específico, concentra-se dentro dos limites setoriais. "A abordagem dos sistemas setoriais de inovação usa uma visão multidimensional, integrada e dinâmica de setores a fim de analisar a inovação" (MALERBA, 2004a: 1).

De acordo com o autor, a noção de SSI "tem origem no conceito de setor, tradicionalmente utilizado na economia industrial" (MALERBA, 2004a: 1), em que "um setor é um grupo de atividades unificadas por algum grupo de produtos relacionados e direcionados a uma dada ou emergente demanda e que compartilham uma base de conhecimento comum. As firmas em um dado setor possuem características comuns e, ao mesmo tempo, são heterogêneas" (MALERBA, 2004b: $385)$.

Franco Malerba descreve e define um sistema setorial com as seguintes palavras:

\footnotetext{
"Assim, um sistema setorial de inovação e produção é composto de um conjunto de produtos novos e estabelecidos para uso específico, e um conjunto de agentes que realizam atividades e interações de mercado e de não-mercado para a criação, produção e venda desses produtos. (...) Os agentes são caracterizados por processos específicos de aprendizagem, competências, crenças, objetivos, estruturas organizacionais e comportamentos. Eles
} 
interagem por meio de processos de comunicação, troca, cooperação, competição e comando, e suas interações são moldadas pelas instituições (regras e regulamentos). No decorrer do tempo, os sistemas setoriais existentes são objetos de diversos processos de mudança e de transformação por meio da co-evolução de seus diversos elementos, e novos sistemas setoriais podem emergir" (MALERBA, 2004a: 16).

Em Wengel e Shapira (2001), é apresentado estudos de caso que relatam os sistemas setoriais de inovação no setor de máquinas e ferramentas em diferentes países e por meio de diferentes prismas. O primeiro caso foca a Alemanha e os relacionamentos encontrados entre fabricantes de máquinas e ferramentas, consumidores, associações, instituições públicas, bem como o desenvolvimento em estrutura e o comportamento das firmas em relação à inovação dentro de seus limites e às tendências tecnológicas externas. O segundo caso examina o sistema setorial de máquinas e ferramentas italiano, focando as tendências estruturais e as razões para uma performance surpreendente nos últimos anos. O terceiro caso descreve a inovação e a estratégia de desenvolvimento sob a perspectiva de uma firma de máquinas e ferramentas japonesa. Finalmente, o foco se dirige para a discussão acerca do desenvolvimento do setor nos Estados Unidos (WENGEL e SHAPIRA, 2001). O foco nos quatro países ocorre em razão dos mesmos serem responsáveis por $67 \%$ da produção mundial do setor - Japão 24\%, Alemanha 21\%, EU 12\%, Itália $10 \%$ - e por $52 \%$ do consumo mundial - EU 21\%, Alemanha 14\%, Japão 10\%, Itália 7\% (WENGEL e SHAPIRA, 2001).

O conceito de sistemas de inovação está relacionado, conforme o termo sugere, a 'sistemas' - trazendo a idéia de complexidade e multiplicidade de conexões - e à 'inovação' - trazendo a idéia de dinamismo e mudanças tecnológicas. A complexidade está relacionada à multiplicidade de atores, às suas características e funções e às conexões existentes, sua intensidade e qualidade. $\mathrm{O}$ dinamismo está relacionado às mudanças e às melhorias tecnológicas, com as quais as firmas se adaptam e se modificam constantemente, buscando a sustentabilidade a longo prazo. É um conceito no qual a característica da localidade e da proximidade física não exerce influências maiores. Sistemas setoriais não possuem limitações geográficas, sendo estudados em termos globais ou em países que possuem destaque no setor.

\section{Considerações finais}

Algumas implicações relativas à concepção desses modelos de análise de aglomerados industriais devem ser levadas em conta. A questão da cultura e da tradição é fundamental para o sucesso de certos tipos de aglomerado. A viabilização de aglomerados industriais, consórcios de firmas e redes de cooperação produtiva não acontece de forma repentina. É um processo lento no qual a cultura e a tradição da atividade devem ser intrínsecas à região e às pessoas envolvidas na 
questão. A concretização dessas configurações está fortemente ligada aos modelos de desenvolvimento regional, à forma de atuação das instituições, às políticas públicas de incentivo e à sociedade como um todo.

No entanto, alguns tipos de aglomeração baseiam-se em oportunidades relativas a recursos naturais (muito comum em setores intensivos em recursos naturais, como o setor de petróleo e gás), ou humano (mão de obra especializada abundante). As empresas permanecem na região apenas em virtude dessas oportunidades. A partir do momento que essas oportunidades não mais existirem, há uma tendência de que essas firmas migrem para outras áreas onde essas oportunidades possam ser exploradas de forma mais intensa. As províncias petrolíferas, baseadas no ciclo de nascimento, crescimento, maturação e declínio, são bons exemplos de aglomeração baseadas em oportunidades específicas.

Como nenhuma empresa, nem mesmo as grandes transnacionais, podem competir sozinhas no mercado, sem nenhum tipo de parceria e cooperação, as abordagens de análise e os conceitos discutidos neste trabalho possuem relevância singular. A empresa que se achar capaz de competir isoladamente estará fadada ao fracasso. A inserção das micro, pequenas e médias empresas na economia moderna passa necessariamente pela proximidade, pela parceria e pelos ganhos competitivos provenientes da cooperação e da inovação.

Com a quebra do monopólio estatal do petróleo, observa-se o interesse e a chegada de grandes petroleiras na região Norte Fluminense. Além disso, cada uma dessas petroleiras traz consigo uma gama de outras empresas fornecedoras de bens e serviços diversos. Essa tendência faz com que o aglomerado se transforme gradativamente de uma estrutura organizacional totalmente verticalizada, no modelo de uma extensa rede de subcontratação, em uma estrutura mais horizontalizada, em que o papel central pode ser dividido entre algumas empresas líderes. Essa mudança pode aumentar a sinergia entre firmas, as chances de alianças e de parcerias estratégicas, o fluxo de conhecimento dentro do aglomerado, as oportunidades para os fornecedores, bem como da capacidade inovadora intrínseca do aglomerado.

Com base na discussão e no contexto apresentados, o arcabouço teórico proposto para a análise do aglomerado de petróleo e gás do Norte Fluminense é focado na interseção da abordagem de cluster e de sistema setorial de inovação.

Assim, são usados alguns elementos de cada uma das abordagens. Da abordagem de cluster, utilizamos o aspecto da aglomeração de firmas em um mesmo espaço geográfico (em uma área relativamente pequena - estado do Rio de Janeiro), sua característica de especialização produtiva, além da estrutura de comando liderada por uma grande empresa (Petrobrás). Da abordagem de sistemas setoriais de inovação, utilizamos o elemento global (sem fronteiras espaciais definidas) e sistêmico do aglomerado. O termo 'sistêmico' introduz a diversidade de atores e a complexidade das 
relações e conexões como duas das principais características. 'Diversidade de atores' é empregado no sentido de analisar cuidadosamente, não somente as firmas, mas também as diversas organizações que exercem papel ativo no desenvolvimento das atividades do aglomerado, tais como: universidades, institutos de pesquisa, organizações reguladoras, organizações públicas, organizações de financiamento, etc. 'Complexidade das relações e conexões' utilizado no sentido de enfatizar as relações e conexões intra- e extra-aglomerado. Atenção especial é dispensada às conexões extra-aglomerado (entre firmas e organizações de dentro do aglomerado e firmas e organizações externas ao aglomerado), o que certamente é pelo menos tão importante quanto as conexões existentes dentro do próprio aglomerado.

\begin{abstract}
Based on the increasing of technological and managerial changes nowadays, firms look for competitive advantages, innovations and the maintenance of their activities in the market in a sustainable way. Aiming to discuss new forms of entering in the new economic dynamic, this paper analyses, under the local productive arrangements (APL's), Japanese keiretsu (Japanese production model), clusters and sectoral innovation systems approaches, pros and cons of these configurations, when used in the analysis of the North Fluminense oil and gas agglomeration. The objective of this paper is identifying the applicability of the concepts and approaches, of the theoretical study, in the North Fluminense oil and gas agglomeration. Moreover, we aim to compare the specificities of some empirical studies, that apply these approaches, with the oil and gas agglomeration in the North Fluminense.
\end{abstract}

Key-words: Innovation; Industrial agglomerations; Oil and gas industry.

\title{
Referências
}

AMAto Neto, J. (2000) - "Redes de Cooperação Produtiva e Clusters Regionais - Oportunidades para as Pequenas e Médias Empresas”- Ed. Atlas, São Paulo - SP.

BEAUDRY, C. \& BRESCHI, S. (2003) - "Are firms in clusters really more innovative?" - Economics of Innovation and New Technologies, Vol. 12(4), pp. 325-342;

cross ref

BELL, M. \& ALBU, M. (1999) - "Knowledge Systems and Technological Dynamism in Industrial Clusters in Developing Countries" - World Development 27 (9): 1715-34;

cross'ref

BERNARDES, R. (2000) - “Redes de Inovação e Cadeias Produtivas Globais: Impactos da Estratégia de Competição da Embraer no Arranjo Aeronáutico da Região de São José dos Campos” - BNDES/FINEP/FUJB, Nota Técnica 23, IE/UFRJ, Rio de Janeiro.

CASSIOLATO, J. E. \& SZAPIRO, M. (2002) - “Arranjos e Sistemas Produtivos e Inovativos Locais no Brasil”Proposição de Políticas para a Promoção de Sistemas Produtivos Locais de Micro, Pequenas e Medias Empresas, UFRJ, Rio de Janeiro, Brasil;

CELESTE, J. L. (1993) - “Especialização Flexível: Uma Alternativa Promissora para o Desenvolvimento Econômico de Regiões Subdesenvolvidas” - Revista de Administração de Empresas (RAE), São Paulo-SP.

CROCCO, M., SANTOS, F., SIMÕES, R. \& HORÁCIO, F. (2001) - “Industrialização Descentralizada: Sistemas Industriais Locais - O Arranjo Produtivo Calçadista de Nova Serrana” - BNDES/FINEP/FUJB, Nota Técnica 36, 
IE/UFRJ, Rio de Janeiro.

GERLACH, M. L.(1992) - “The Keiretsu: a primer” - New York: Japan Society.

LAstres, H. M. M. \& CASSiOlato, J. E.(2000) - “A Política Tecnológica Japonesa” - Instituto de Pesquisa de Relações Internacionais, Seminário sobre o Japão, Rio de Janeiro - RJ.

LA ROVERE, R. L., HASENCleVER, L., MElO, L. M., FIALHO, B. C. \& SilVA, M. M. (2000) “Industrialização Descentralizada: Sistemas Industriais Locais Estudo do Setor Têxtil e de Confecções” BNDES/FINEP/FUJB, Nota Técnica 15, IE/UFRJ, Rio de Janeiro.

MALERBA, F. (Ed.) (2004a) - "Sectoral Systems of Innovation - Concepts, Issues and Analyses of Six Major Sectors in Europe" - Cambridge University Press;

MALERBA, F. (2004b) - "Sectoral Systems - How and Why Innovation Differs Across Sectors" - in FAGERBERG, J., MOWERY, D. C. \& NELSON, R. R. (Eds.) (2004) - “The Oxford Handbook of Innovation” - Oxford Handbooks;

MALMBERG, A. (2003) - “Beyond the Cluster - Local Milieus and Global Connections”- in PECK, J. \& YEUNG, W. (Eds.) (2003) - "Remaking the Global Economy - Economic-Geographical Perspectives" - SAGE;

MASIERO, G. (1995) - "Principais Aspectos da Administração Japonesa" - Revista de Administração de Empresas, ERA/EAESP/FGV, São Paulo, SP.

PORTER, M. E. (1998) - "Clusters and the New Economics of Competition" - Harvard Business School, NovemberDecember;

RANGEL, J. (2005) - “Petrobrás lucra R\$ 5 bilhões no $1^{\circ}$ trimestre” - Jornal O Globo de Sábado, 14 de maio de 2005, Caderno Economia, p. 31;

SAXENIAN, A. (1994) - “Regional Advantage. Culture and Competition in Silicon Valley and Route 128”Cambridge, MA, Harvard University Press;

SCHMITZ, H. (1997) - “Collective Efficiency and Increasing Returns” - IDS working Paper 50, University of Sussex, Brighton, UK;

SCHMITZ, H. (1999) - "Global Competition and Local Cooperation: Success and Failure in the Sinos Valley, Brazil”World Development Vol. 27 (9), pp. 1627-1650;

cross'

Silvestre, B. S. \& DAlCOL, P. R. T. (2004) - “O Setor de Petróleo do Rio de Janeiro: Arranjo Produtivo Local ou Modelo dos Keiretsu de Produção Japoneses” - EnEO 2004, Atibaia - SP.

SILVESTRE, B. S. \& DALCOL, P. R. T. (2003) - “Redes de Empresas e Arranjos Produtivos Locais: Alternativas para Inserção de Empresas na Nova Economia” - XXVII ENANPAD, Atibaia - SP.

VARgas, M. A. \& ALIEVI, R. M. (2000) - “Arranjo Produtivo Coureiro-Calçadista do Vale dos Sinos/RS”BNDES/FINEP/FUJB, Nota Técnica 21, IE/UFRJ, Rio de Janeiro.

VILLASCHI, A. F. \& SABADINI, M. S. (2000) - “Arranjo Produtivo de Rochas Ornamentais (mármore e granito) no estado do Espírito Santo” - BNDES/FINEP/FUJB, Nota Técnica 15, IE/UFRJ, Rio de Janeiro.

WENGEL, J. \& SHAPIRA, P. (2001) - “Machine Tools: The Remaking of aTraditional Sectoral Innovation System?" - Sectoral Systems in Europe - Innovation, Competitiveness and Growth (ESSY, Targeted Socio-Economic Research, TSER;

WOMACK, J. P., JONES, D. T. \& ROSS, D. (1992) - “A Máquina que Mudou o Mundo” - Ed. Campus, Rio de Janeiro, RJ.

Nome completo: Bruno dos Santos Silvestre

Filiação institucional: Centrais Elétricas Brasileiras S. A. - ELETROBRÁS

Departamento: Departamento de Novos Negócios

Função ou cargo ocupado: Supervisor de Negócios

Endereço completo para correspondência (bairro, cidade, estado, país e CEP): Rua Álvaro Tâmega - 29, Centro, Campos dos Goytacazes - RJ, CEP: 28035-030; Brasil. 
Telefones para contato: (22) 9811-6645; (21) 2514-6064

e-mail: bruno.silvestre@eletrobras.com ou bruss@ind.puc-rio.br

Nome completo: Paulo Roberto Tavares Dalcol

Filiação institucional: Pontifícia Universidade Católica do Rio de Janeiro - PUC-Rio

Departamento: Departamento de Engenharia Industrial - DEI

Função ou cargo ocupado: Professor Associado

Endereço completo para correspondência (bairro, cidade, estado, país e CEP): Rua Marquês de São

Vicente, 225 - Gávea, Rio de Janeiro, RJ, CEP: 22453-900

Telefones para contato: (21) 3527-1284, 3527-1285

e-mail:prtd@ind.puc-rio.br

Recebido para publicação em: 03/05/07

Aceito para publicação em: 01/06/07 